\title{
USO PEDAGÓGICO DEL BLOG: UN PROYECTO DE INVESTIGACIÓN-ACCIÓN EN LA MATERIA DE EDUCACIÓN FÍSICA EN EDUCACIÓN SECUNDARIA
}

\author{
PEDAGOGICAL USE OF THE BLOG: AN ACTION-RESEARCH PROJECT IN \\ PHYSICAL EDUCATION AT THE SECONDARY LEVEL
}

\author{
Luis Santos Rodríguez; luis.santos.ef@gmail.com \\ Consejería de Educación de Castilla y León \\ Javier Fernández-Río; javier.rio@uniovi.es \\ Universidad de Oviedo
}

\section{RESUMEN}

El objetivo de este trabajo fue conocer los efectos del uso del blog como recurso de apoyo a la materia de Educación Física. 108 estudiantes de 4으 de ESO participaron en la experiencia, desarrollada durante un curso escolar. Los datos se recogieron mediante un cuestionario desarrollado ad hoc. También se realizaron entrevistas semi-estructuradas para obtener más información. Los resultados indican el gran valor pedagógico que los estudiantes dan a estas herramientas digitales.

PALABRAS CLAVE: Sistema Educativo, material, apoyo.

\section{ABSTRACT}

The goal of this project was to assess the effects of the use of a blog as a resource in Physical Education. 108 students of $4^{\text {th }}$ grade of Secondary Education participated in the project, which lasted a complete academic year. Data was collected via questionnaire. Semistructured interviews were also conducted to obtain more information. Results show the high pedagogical value that students give to these digital tools.

KEY WORDS: Educational System, resource, support. 


\section{INTRODUCCIÓN}

Las Tecnologías de la Información y la Comunicación (TIC) están cada vez más presentes en nuestra sociedad. En la actualidad, la oleada tecnológica es de tal magnitud que está afectando a la manera de trabajar de todos los sectores profesionales.

El Sistema Educativo debe responder, y al mismo tiempo anticiparse, a los acontecimientos, dotando al alumnado de las competencias tecnológicas y digitales necesarias. La pedagogía digital debe orientarse hacia la adquisición y el desarrollo de las habilidades del aprendizaje autónomo, de comunicación, de investigación, de análisis y de síntesis.

La materia de Educación Física ni puede, ni debe, quedar al margen de esta situación por lo que sus docentes se ven obligados, cada vez más, a desarrollar una verdadera ingeniería pedagógica para contribuir, en la mayor medida posible, al proceso de formación integral del individuo impulsado por el Sistema Educativo vigente. De hecho, ya hay voces (Rodera Bermúdez, 2008) que señalan un creciente interés de los docentes de esta área por introducir las TIC en el aula de Educación Física, sobre todo a través de las webquest (Dodge, 1995).

Una de las herramientas digitales más populares en todo el mundo son los denominados blogs, que son páginas web muy sencillas donde el usuario puede publicar comentarios, artículos, fotografías, enlaces o vídeos (Bohórquez Rodríguez, 2008). La bibliografía especializada recoge trabajos que muestran las virtudes del uso pedagógico del blog en la enseñanza no universitaria en materias curriculares como Historia, Literatura o Civismo (Drexler, Dawson \& Ferdig, 2007; De Gennaro \& Brown, 2009) para fomentar el aprendizaje cooperativo (Drexler, Dawson \& Ferdig, 2007), la motivación para leer y escribir (AmorósPoveda, 2007), la autoestima de sus usuarios (Nagel \& Anthony, 2009), el intercambio de ideas, el trabajo en equipo, la capacidad de síntesis, la expresión escrita, la creatividad, la comunicación o la iniciativa (Cuesta y Gómez, 2008; Martín y Alonso, 2009).

En el ámbito de la enseñanza universitaria, Pérez et al. (2012) afirman que el uso adecuado de los blogs parece de gran utilidad para fomentar una enseñanza más colaborativa y participativa. Sin embargo, estos mismos autores manifiestan que a pesar de las muchas posibilidades que tienen los blogs para mejorar el proceso educativo, su uso no está demasiado extendido.

Todo lo anterior nos llevó a plantear y desarrollar una experiencia real de investigaciónacción sobre el uso pedagógico del blog dentro de la Educación Física. Así, los principales objetivos de este trabajo eran: por un lado, conocer los efectos sobre los estudiantes del uso del blog como material de refuerzo de la materia de Educación Física en la en Educación Secundaria Obligatoria (ESO) y, por otro lado, evaluar el potencial pedagógico de dicha herramienta.

Este trabajo de investigación-acción se estructura del siguiente modo. En primer lugar, se despliega una fundamentación teórica sobre el uso del blog en el ámbito educativo. En segundo lugar, se realiza una descripción de la experiencia basada en la utilización del blog en el proceso de enseñanza-aprendizaje. En tercer lugar, se desarrolla la metodología utilizada en la investigación. En cuarto lugar, se exponen los resultados obtenidos. Por 
último, se discute lo encontrado y se señalan las principales conclusiones e implicaciones que se derivan de la experiencia y del estudio realizado.

\section{EL BLOG COMO HERRAMIENTA EDUCATIVA DE PRIMER ORDEN}

La elección del blog dentro de la oferta de los diferentes recursos digitales (portal web, redes sociales...) como material de apoyo y refuerzo de una materia curricular (en este caso, Educación Física) se apoya en los diferentes estudios presentados en el apartado anterior, pero además, en la realidad de que los blogs son un fenómeno en constante crecimiento en nuestra sociedad cuyo uso se ha masificado (Blood, 2006). Hasta el año 2009 se habían registrado en todo el mundo más de 200 millones de blogs (Torres Zuñiga, 2009); sin embargo, aquellos dedicados a ciencia y educación eran muchos menos en comparación con los de vida y estilo. Igualmente, los blogs escritos en español tienen una representación muy pequeña: 3\%. Es decir, son muy pocos los blogs dedicados a temas de ciencia y educación y escritos en español.

Bohórquez Rodríguez (2008) plantea otras virtudes que convierten al blog en una herramienta de apoyo y refuerzo de las materias del sistema educativo:

- Alojamiento gratuito en Internet (no hay ningún coste).

- Posibilidad de incluir contenidos muy diversos (expande su impacto).

- Acceso libre (aunque existe la posibilidad de restringir el acceso).

- Administración muy sencilla (facilita la labor del docente).

- Posibilidad de que los usuarios participen (a través de comentarios).

Díaz Huerta (2009) afirma que el blog es un recurso muy valioso de apoyo a la clase presencial y para la generación de conocimiento, ya que tiene como medio de transmisión Internet, que es un medio de comunicación cotidiano para los jóvenes y éstos lo aceptan como vehículo de aprendizaje gracias a sus virtudes: libertad, flexibilidad e informalidad. Este autor mantiene que el blog permite desarrollar una comunicación maestro-alumno más estrecha y de mayor confianza (más horizontal), que provoca mayor apertura y libertad para expresar pensamientos, sentimientos, dudas e inquietudes.

Por otro lado, las redes sociales (Facebook, Twitter...) que inundan Internet, podrían ser herramientas valiosas en el ámbito educativo. No obstante, Nielsen (2006) señala que en estas comunidades el $1 \%$ de usuarios generan contenido y son muy participativos, el $9 \%$ publican algunos comentarios y un $90 \%$ se limita a observar. Por el contrario, los blogs parecen propiciar una mayor participación de los usuarios. El objetivo de toda herramienta educativa debe ser conseguir que la mayor parte del alumnado se comporte de manera activa generando contenidos, trabajos, ideas... Por ello, uno de los objetivos de este proyecto era que los estudiantes participantes se convirtieran en usuarios activos del blog.

Rodera Bermúdez (2008) considera que tres son los ámbitos fundamentales que deben considerarse a la hora de diseñar y utilizar herramientas digitales en el contexto educativo. A continuación mostramos como fueron contemplados en nuestra experiencia y para nuestra herramienta: 
- Pedagógico: incluyendo materiales adecuados para el desarrollo de la asignatura: apuntes de las distintas unidades didácticas, ejercicios prácticos de refuerzo, documentos del Plan de Fomento a la Lectura...

- Técnico: empleando aplicaciones informáticas útiles e innovadoras como el programa Hot Potatoes para plantear exámenes a través del blog o la aplicación informática Voicethread para realizar comentarios de audio.

- Estético: diseñando y manteniendo un formato atractivo para los usuarios (modificamos periódicamente el diseño del blog a partir de las propuestas de los estudiantes incluyendo fotos, videos o música en los textos escritos...).

\section{DESCRIPCIÓN DE LA EXPERIENCIA}

Para desarrollar esta experiencia de investigación-acción diseñamos un plan de trabajo que se concretó en tres pasos fundamentales.

1. Selección de los sujetos participantes. El objetivo era trabajar con una población de estudiantes con el nivel madurativo adecuado como para comprometerse a participar en una investigación de estas características. Además, dicha población debía ser lo suficientemente cuantiosa como para obtener resultados significativos. Así, todos los estudiantes de los cuatro grupos de 40 de la ESO de un centro de Enseñanza Secundaria participaron en la investigación. Tanto el equipo directivo del centro educativo, como el equipo docente de los grupos seleccionados avalaron la idoneidad de dicho grupo de estudiantes para participar en una investigación de estas características. Los indicadores en los que se basó dicho aval fueron: calificaciones, número de incidencias de comportamiento, faltas de asistencia y grado de participación en eventos educativos de carácter voluntario (semanas culturales, talleres pedagógicos...). Respecto al centro educativo donde se llevó a cabo la experiencia, éste puede considerarse plenamente urbano en el que el nivel socioeconómico del alumnado es medio.

2. Elección de una plataforma digital adecuada para albergar un blog de carácter pedagógico. Antes de diseñar, elaborar y activar el blog como material de apoyo y refuerzo de la materia de Educación Física necesitábamos escoger una plataforma adecuada para alojarlo en internet. Actualmente, las más importantes son Blogger, Bitacoras.com y WordPress. Tras muchas consultas a diferentes especialistas, decidimos usar Blogger por dos motivos: (a) la estabilidad y fiabilidad tecnológica de esta plataforma (para evitar posibles fallos de funcionamiento que pudieran poner en riesgo la investigación) y (b) la sencillez en la configuración, diseño y mantenimiento de la misma. El nombre escogido para el blog fue: laeducacionfisicadigital.blogspot.com.

3. Diseño y puesta en práctica de la experiencia a lo largo de un curso académico completo. Los elementos sobre los que quisimos incidir a través de nuestra propuesta estaban directamente relacionados con el Decreto 52/2007 que establece el Currículum de la ESO en Castilla y León (Consejería de Educación de Castilla y León, 2007). A través del blog quisimos incidir en elementos curriculares de tres materias 
diferentes del 4으 curso de la ESO. Educación Física: el proyecto tenía como base fundamental dicha materia por lo que los contenidos del blog hacían referencia a ella, la actividad física y al Deporte. Informática: ya que según se expone en el Decreto, ésta ha ido adquiriendo una importancia progresiva en la vida de las personas y en el funcionamiento de la sociedad; sin embargo, muchas aún no la han incorporado a su vida diaria; por ello, la inclusión de herramientas como el blog en el sistema educativo resulta de vital importancia para la formación de las personas (Consejería de Educación de Castilla y León, 2007). Tecnología: ya que el Decreto afirma que la aceleración que se ha producido en el desarrollo tecnológico durante el siglo XX justifica la necesidad formativa en este campo. El ciudadano (y por extensión el alumnado) precisa de conocimientos suficientes para ser un agente activo en este proceso, ya sea como consumidor de los recursos que la tecnología pone en sus manos o como productor de innovaciones. El uso de blogs fomenta la adquisición de conocimientos y el desarrollo de destrezas que permiten tanto la comprensión de los objetos técnicos como su utilización (Consejería de Educación de Castilla y León, 2007). Este Decreto pretende también que el alumnado use las TIC como herramientas en este proceso y no como fin en sí mismas. En base a ello, los objetivos fundamentales del uso del blog en la presente experiencia fueron:

1. Familiarizar al alumnado con las Nuevas Tecnologías y motivarle para que las utilice en su vida cotidiana.

2. Desarrollar y facilitar el acceso de todos los contenidos de la Educación Física de forma novedosa y motivante.

3. Fomentar el hábito de la lectura del alumnado desde las TIC.

4. Generar una vía de comunicación entre profesorado, alumnado y familias.

5. Servir como recurso a programas educativos como el Comenius.

6. Ofrecer algo "nuevo" al alumnado, que le sorprenda positivamente en su formación, que despierte su interés por aprender y esforzarse cada día.

La metodología básica de trabajo del blog se concretó en la activación de diferentes enlaces para descargar archivos (apuntes, materiales prácticos, lecturas, artículos, secuencias de video, exámenes, cuestionarios de evaluación...). El objetivo era que los estudiantes tuvieran un fácil y rápido acceso a todos los materiales, así como a la realización de las tareas que se proponían y que debían ser publicadas en el blog como "comentarios" en entradas específicas para cada una de ellas. Así, nuestra propuesta de uso del blog en la Educación Física aspiraba a:

- Plantear tareas de Educación Física de manera no-presencial, desarrollando el concepto de "deberes" en ésta.

- Registrar parte del trabajo que el alumnado desarrolla para que las familias puedan seguirlo desde sus hogares.

- Evitar cargar al alumnado con libros o documentos (todos los materiales eran online).

Las actividades de enseñanza-aprendizaje que el docente planteaba a través del blog fueron fundamentalmente tres: 
a) Tareas propias de cada unidad didáctica: lectura reflexiva de artículos periodísticos (pudiendo expresar en "comentarios", las reacciones correspondientes), cuestionarios de autoevaluación, visionado de secuencias de video (con la posibilidad de hacer "comentarios")...

b. Pruebas escritas o tests: para que alumnado, profesorado y familias tuvieran acceso inmediato a los resultados.

c. Información y/o documentación relevante: fechas de exámenes, avisos de actividades extraescolares o complementarias, apuntes, lecturas, autorizaciones...

- Además, el alumnado, a través del blog, podía participar en el proceso de enseñanza-aprendizaje:

- Diseñando y proponiendo parte del trabajo a realizar en sesiones futuras (los compañeros podían opinar sobre la conveniencia o no de su introducción).

- Proponiendo unidades didácticas relacionadas con los objetivos de la materia.

- Publicando "comentarios" de aspectos interesantes o que necesitasen ser revisados.

- Creando foros de debate.

- Proponiendo la publicación de enlaces de noticias, videos, webquest....

- Compartiendo con estudiantes de otros países a través del proyecto Comenius.

- Proponiendo la publicación de fotografías o videos de actividades extraescolares y/o complementarias relacionadas con la asignatura.

\section{METODOLOGÍA}

Un total de $\mathrm{N}=108$ estudiantes (44 varones y 64 mujeres), cuya edad media era de 15,32 años, participaron en esta experiencia de investigación-acción que se desarrolló a lo largo de un curso escolar completo.

Para evaluar los resultados de la experiencia desarrollada, todos los sujetos cumplimentaron una encuesta anónima desarrollada ad hoc, a través de la que se perseguía conocer sus opiniones sobre el uso del blog en la materia de Educación Física.

Posteriormente, se realizaron entrevistas semi-estructuradas a un $75 \%$ de los estudiantes participantes. El objetivo era obtener información más concreta de la opinión del alumnado acerca de la experiencia. Para ello, se utilizaron las preguntas del cuestionario, a las cuales se añadió alguna pregunta más para profundizar en las opiniones de los estudiantes. Las respuestas obtenidas fueron analizadas usando el método de comparación constante (Lincoln \& Guba, 1985) y el de inducción analítica (Patton, 1990) con objeto de identificar y extraer categorías y patrones de respuesta comunes. Primeramente, se transcribieron las entrevistas y éstas fueron leídas por dos investigadores. Éstos, por separado, establecieron 
las categorías a partir del análisis y agrupamiento de las distintas respuestas. Identificadas las categorías de análisis, éstas se compararon, encontrando un índice de acuerdo interobservador de un $85 \%$. A continuación, los datos fueron re-analizados con el objetivo de encontrar discrepancias o malas interpretaciones (Miles \& Huberman, 1994). Este proceso implicó a dos investigadores que contrastaron de forma conjunta que las categorías iniciales pre-establecidas coincidían con las encontradas tras el análisis.

\section{RESULTADOS}

\section{Cuestionario}

La tabla 1 muestra las respuestas del alumnado participante sobre del uso del blog en el contexto educativo. Algunas de las preguntas se centraban en aspectos generales, mientras que la mayoría intentaban obtener información de los sujetos tras una experiencia pedagógica del uso del blog asociado a la materia de Educación Física durante un año académico completo.

A nivel general, el 98,8\% de los estudiantes participantes manifestó poseer conexión a Internet en sus hogares. El 99,5\%, consideraba adecuado el uso del blog en el sistema educativo y el $96,7 \%$ opinaba que el uso de éste dentro del sistema educativo ayuda a mejorar el conocimiento teórico-práctico de las TIC.

Las siguientes preguntas pretendían hacer hincapié en las opiniones del alumnado acerca del uso, dentro de la materia de Educación Física, del blog denominado: "laeducacionfisicadigital.blogspot.com". Así, el 98,6\% del alumnado consideró adecuada la forma en la cual se desarrollaron los exámenes de la materia a través del blog. El 97,7\% estimó adecuada la manera de realizar las tareas del Plan de Fomento a la Lectura a través del blog. El 97,8\% consideró adecuada la manera en la cual el blog permitía el acceso a los apuntes de la materia. Al $98,1 \%$ le parecieron interesantes y didácticos los ejercicios prácticos del blog como actividades de refuerzo de las unidades didácticas desarrolladas. Finalmente, al 85,8\% del alumnado le resultó suficientemente sencillo el uso del blog.

Tan sólo hubo un apartado que no produjo resultados tan espectacularmente positivos: sólo el $67,2 \%$ del alumnado juzgó interesantes los artículos periodísticos publicados en la página principal del blog. 


\begin{tabular}{|l|c|c|}
\hline \multicolumn{1}{|c|}{ CUESTIONES } & SI & NO \\
\hline 1. ¿'Dispones de conexión a Internet en tu hogar? & $98,8 \%$ & $1,2 \%$ \\
\hline 2. ¿Te parece adecuado el uso del blog en el sistema educativo? & $99,5 \%$ & $0,5 \%$ \\
\hline $\begin{array}{l}\text { 3. ¿El uso del blog en el sistema educativo ayuda a mejorar el conocimiento } \\
\text { teórico-práctico de las TIC? }\end{array}$ & $96,7 \%$ & $3,3 \%$ \\
\hline $\begin{array}{l}\text { 4. ¿Te parece adecuada la forma en la cual se desarrollan los exámenes de } \\
\text { la materia a través del blog? }\end{array}$ & $98,6 \%$ & $1,4 \%$ \\
\hline $\begin{array}{l}\text { 5. ¿'Te parece adecuada la manera de realizar las tareas del Plan de } \\
\text { Fomento a la Lectura a través del blog? }\end{array}$ & $97,7 \%$ & $2,3 \%$ \\
\hline $\begin{array}{l}\text { 6. ¿Te parece adecuada la manera en la cual el blog te permite el acceso a } \\
\text { los apuntes de la materia? }\end{array}$ & $97,8 \%$ & $2,2 \%$ \\
\hline $\begin{array}{l}\text { 7. ¿Te parecen interesantes y didácticos los ejercicios prácticos del blog } \\
\text { como actividades de refuerzo de las unidades didácticas? }\end{array}$ & $98,1 \%$ & $1,9 \%$ \\
\hline $\begin{array}{c}\text { 8. ¿Te resultan interesantes los artículos periodísticos publicados en el } \\
\text { blog? }\end{array}$ & $67,2 \%$ & $32,8 \%$ \\
\hline 9. ¿'Te resulta suficientemente sencillo el uso del blog? & $85,8 \%$ & $14,2 \%$ \\
\hline
\end{tabular}

Tabla 1. Resultados del cuestionario de evaluación del uso pedagógico del blog en la Educación Física en la ESO

\section{Entrevistas}

En la presentación de resultados de esta parte de la investigación hemos optado por seguir el hilo conductor de las cuestiones planteadas en las entrevistas.

1. ¿Te parece adecuado el uso del blog en el sistema educativo? La categorización de los resultados obtenidos muestran estos términos: innovación, diversión, comodidad, practicidad, rapidez, sencillez y ahorro.

"Sí, porque es otra manera de hacer trabajos y aprender a utilizar las nuevas tecnologías" (Ana)

"Sí, siempre resulta más interesante e innovador utilizar otros métodos y éste crea gran interés" (Isabel)

2. ¿El uso del blog dentro del sistema educativo ayuda a mejorar el conocimiento teórico-práctico de las TIC? Los resultados categorizados señalan vocablos como: promueve su uso, motivación, divertido, práctico y fácil.

"Sí porque sin darte cuenta practicas con las nuevas tecnologías" (Pedro)

"Sí, ya que se demuestra que no es sólo para algo de ocio, tiene muchas aplicaciones más" (Beatriz)

3. Los exámenes de la materia se realizan a través del blog, ¿qué te parece? Esta pregunta tan comprometida generó las siguientes categorías de respuestas: rapidez, sencillez, motivación, conocimiento de resultados y ahorro. 
"Sí, son más rápidos y obtienes la corrección al momento" (Álvaro)

"Sí, me parece adecuado ya que ahorramos papel y nos relacionamos con las nuevas tecnologías" (Cecilia)

4. Con respecto al Plan de Fomento a la Lectura, ¿qué te parecen los textos que se recogen en el blog? La categorización de las respuestas emitidas por los sujetos participantes dio como resultado los siguientes vocablos: interesantes, entretenidas, diferentes, didácticas y ahorro.

"Es menos cansado y entretenido de esta forma" (Ana)

"Nos da una mejor capacidad para resumir y condensar la ideas principales en un texto" (Andrés)

5. Con respecto al Plan de Fomento de la Lectura, ¿te parecen adecuadas las tareas propuestas? Los resultados obtenidos fueron agrupados en las siguientes categorías: interesantes, consolidan conocimientos y prácticas.

"Sí, son más interesantes estos artículos o relatos narrados que leerte un libro" (Isabel)

"Me parece muy bien porque de esta manera ampliamos nuestros conocimientos de manera más concreta sin tener que estudiar tanto" (Elvira)

6. El blog digital te permite el acceso a los apuntes de la materia, ¿qué te parece? Revisadas las respuestas de los estudiantes, las categorías emergentes han sido: disponibilidad, accesibilidad, innovación, ahorro de papel y de espacio.

"Están más disponibles, se ahorra papel y además si se pierden se pueden volver a descargar" (Andrea)

"Yo pienso que está muy bien innovar y hacerlo todo por Internet, ya que dentro de unos años todo será por ahi" (Leticia)

7. ¿Qué te parecen los ejercicios prácticos publicados en el blog como actividades de refuerzo de las unidades didácticas? Las respuestas emitidas a esta pregunta generaron las siguientes categorías: sencillez, rapidez, diversión, ayuda a la comprensión, refuerzo de conocimientos.

"Son ejercicios sencillos y rápidos que ayudan a reforzar los conocimientos adquiridos" (Marta)

"Todo lo que nos ayude a comprender las cosas está bien" (Elizabeth)

8. ¿Qué te parecen los artículos periodísticos publicados en la página principal del blog? La categorización de las respuestas dio como consecuencia estos términos: interesantes, innovadores, consolidan o amplían conocimientos.

"Son muy interesantes y aportan algo muy importante, como es algo de cultura general" (Pedro) 


\section{"Así adquiero conocimientos nuevos" (Enrique)}

9. Con respecto al uso del blog, ¿te resulta sencillo o por el contrario se deberían introducir cambios? Los términos más repetidos en las respuestas de los estudiantes pueden agruparse en: sencillez, claridad, organización.

"Es sencilla y fácil de entender" (Jorge)

"Me parece fácil de entrar y de encontrar los contenidos" (María)

10. ¿Podrías sugerir algún tipo de mejora en el blog? Las ideas aportadas por los estudiantes se podrían agrupar en: música, color, fotos, videos, esquemas de los recursos y aviso de nuevas entradas vía sms o e-mail.

"Poner esquemas porque de esta forma podemos elaborar resúmenes de la materia" (Rita)

"Alguna mejora como poner música, darle más color y cambiar el diseño" (Adolfo)

Aunque una abrumadora mayoría de las respuestas en todas las preguntas fueron positivas (ver tabla 1), también hubo un porcentaje de respuestas negativas. Como éstas han sido poco numerosas, en lugar de analizar y presentar los resultados cuestión por cuestión, como en el caso de las respuestas positivas, hemos hecho una categorización global de todas las respuestas. De dicho proceso hemos obtenido los siguientes conceptos: dificultad y duplicidad.

"Una persona inexperta no puede manejar estos ordenadores" (Pedro)

"Prefiero la manera tradicional ya que el papel lo puedes llevar a cualquier lugar para estudiar y en cambio desde el ordenador necesitas conexión a Internet y no hay en todo los sitios" (Marta)

"Para ello ya existe otra asignatura llamada Lengua Castellana y Literatura" (Ana)

"Creo que todos tenemos suficientes conocimientos sobre las nuevas tecnologías" (José)

Finalmente, consideramos interesante presentar datos adicionales que pueden dar una idea del grado de uso que los participantes hicieron de esta herramienta de trabajo: se realizaron 543 comentarios en el blog y éste tuvo más de 5.237 visitas (aunque más de 1.000 de ellas se localizaron fuera de España; es decir, no fueron realizadas por nuestro alumnado). El 26,8\% de los estudiantes participantes se hicieron seguidores permanentes del blog y el $99,5 \%$ lo tienen adscrito a su selección de webs favoritas en su ordenador. Por último, 3 de los estudiantes participantes han activado un blog en la red.

\begin{tabular}{|l|c|}
\hline Número de comentarios realizados & 543 \\
\hline Número de visitas al blog & 5.237 \\
\hline Seguidores permanentes del blog & $26,8 \%$ \\
\hline Adscrito como webs favoritas & $99,5 \%$ \\
\hline Blogs activados & 3 \\
\hline
\end{tabular}

Tabla 2. Otros datos sobre los efectos del uso del blog 
Al mismo tiempo, también queremos señalar que el 95,3\% del alumnado participante desarrolló, en algún momento, las tareas propias del blog. La frecuencia trimestral media de acceso al blog por parte de cada estudiante se situó en 4,63 visitas por unidad didáctica durante el primer trimestre, y durante el segundo y el tercero alcanzó las 3,89 visitas. Finalmente, a lo largo de los meses estivales, un porcentaje del $21,8 \%$ del alumnado participante continuó visitando el blog para acceder a sus contenidos.

\section{DISCUSIÓN}

Lo primero que nos gustaría destacar es el dato de que prácticamente la totalidad del alumnado participante $(98,8 \%)$ disponía de conexión a Internet en sus casas. Esta circunstancia constituyó un excelente punto de partida para poder garantizar el éxito de la investigación (cuales quiera que fueran los resultados obtenidos) y dar mayor validez a las respuestas de los participantes en el proyecto.

En la actualidad, sigue activo el debate de cómo convertir a las TIC en un aliado de la formación reglada, ocupacional y continua (Rodera Bermúdez, 2008). El presente estudio muestra una forma de abordar este objetivo en la ESO y en una asignatura considerada muy "práctica", y alejada de las TIC, como la Educación Física. Los resultados ponen de manifiesto que los estudiantes consideran que el uso del blog contribuye a la adquisición y al desarrollo de la Competencia Básica número 5: Tratamiento de la Información y Digital, prescrita por las leyes educativas vigentes. Por tanto, el uso pedagógico del blog se revela como un estimulo para aprender a encontrar, organizar y compartir información entre el alumnado. Además, siguiendo a Bohórquez Rodríguez (2008), dicha información puede ser comentada, con lo que el alumnado puede obtener retroalimentación.

Así mismo, el alumnado participante considera de manera abrumadora que el blog es una herramienta fácil de utilizar, práctica y rápida, que ayuda a consolidar y ampliar los conocimientos previos, que ahorra tiempo, espacio y papel y que proporciona una gran accesibilidad a los materiales de la asignatura. Todas estas características hacen que sea una herramienta innovadora y motivante que permite organizar con claridad los contenidos y tenerlos a plena disposición en todo momento. Así mismo, facilita la conexión entre los docentes, los discentes y las familias, lo que ayuda a fortalecer la confianza mutua durante todo el proceso de enseñanza-aprendizaje.

Algunas de las conclusiones a las que llegó Torres Zuñiga (2009), en un estudio con alumnado universitario, están en la línea de los razonamientos que hemos desarrollado a la luz de los resultados de nuestra investigación, ya que afirma que los blogs son una vía prometedora de creación de clases transversales, critica constructiva y trabajo colaborativo.

Las respuestas de los estudiantes participantes en nuestra experiencia podrían animar al profesorado de ésta y otras materias a acercarse a las TIC para incluirlas como herramientas pedagógicas en su desempeño profesional. Como señalan Martínez Sánchez y Prendes Espinosa (2003), en la mayoría de las ocasiones, los docentes nos acercamos a la tecnología con miedo, incertidumbre y con gran escepticismo, lo que hace que no aprovechemos todas sus posibilidades. Nuestros resultados indican que los estudiantes valoran esta herramienta muy positivamente, por lo que los docentes deberían hacer uso de ella. 
Así mismo, hemos podido comprobar la importancia que los parámetros pedagógicos, técnicos y estéticos de los blogs tienen en el uso pedagógico de éstos como material de apoyo y refuerzo de una materia educativa. Propuestas como esquemas orientativos en los contenidos, música, más color, fotos y videos o avisos a través de sms o e-mails de nuevas entradas constituyen valiosas sugerencias de los estudiantes para mejorar esta herramienta educativa. Por eso, los administradores de los blogs pedagógicos, que no son otros que los docentes, deberían prestar atención a estos tres aspectos, ya que ellos van a determinar el éxito pedagógico del blog.

Sin embargo, estamos totalmente de acuerdo con la reflexión de Rodera Bermúdez (2008, p. 4); "lo realmente relevante no son las Nuevas Tecnologías en sí mismas, sino el uso que hagamos de ellas". Efectivamente, las TIC ni sirven para todo, ni van a solucionar todos los problemas pedagógicos a los que deben enfrentarse diariamente los docentes, sino que son un recurso didáctico más, que puede enriquecer significativamente la ingeniería pedagógica que el profesorado debe desarrollar para alcanzar los objetivos que se ha planteado, tanto en lo que se refiere a los aspectos académicos (Competencias Básicas) como a aquellos que atañen al desenvolvimiento en el ambiente cotidiano.

Como futuras líneas de investigación, proponemos extender esta experiencia pedagógica al resto de niveles de la ESO y al Bachillerato, y posteriormente, con las necesarias adaptaciones, a la Etapa de Educación Primaria y a los programas de enseñanza bilingüe. En este último caso, debido al elevado número de blogs existentes en inglés, podría resultar interesante desarrollar experiencias pedagógicas de investigación, como la que se recoge en este documento, en las secciones bilingües de enseñanzas no universitarias.

Así mismo, sería interesante abrir la herramienta a las familias para que padres y madres puedan participar, al menos en parte, en el diseño, puesta en escena y desarrollo de la asignatura.

\section{CONCLUSIONES}

Los resultados obtenidos en el presente trabajo ponen de manifiesto que el blog puede ser considerado y/o utilizado como material de apoyo y refuerzo en la ESO. Asimismo, el diseño, la estructura, los contenidos y la operatividad del blog denominado: laeducacionfisicadigital.blogspot.com se han revelado como apropiados para su uso pedagógico dentro de la materia de Educación Física.

\section{REFERENCIAS}

AMORÓS-POVEDA, L. (2007). Diseño de weblogs en la enseñanza. EDUTEC, Revista Electrónica de Tecnología Educativa, 24. Consultado el 20/03/12. http://edutec.rediris.es/Revelec2/revelec24/lamoros/lamoros.htm

BLOOD, R. (2006). Weblogs: A History and Perspective. En el sitio web Rebecca's Pocket. Generado el 25/10/2006. Consultado el 29 de febrero de 2012. http://www.rebeccablood.net/essays/webloghistory.html. 
BOHÓRQUEZ RODRíGUEZ, E. (2008). El blog como recurso educativo. Edutec, Revista Electrónica de Tecnología Educativa, 26. Consultado el 14/04/12. http://edutec.rediris.es/Revelec2/Revelec26/

CONSEJERÍA DE EDUCACIÓN DE CASTILLA Y LEÓN. (2007). Decreto 52/2007 de 17 de mayo, por el que se establece el Currículo de la Educación Secundaria Obligatoria en Castilla y León.

CUESTA, P. y GÓMEZ, A.M. (2008). Web 2.0 y Educación. Revista de Formación e Innovación Educativa Universitaria, 1(2), 52-57.

DE GENNARO, D. \& BROWN, T.L. (2009). Youth voices: connections between history, enacted culture and identity in a digital divide initiative. Cultural Studies of Science Education, 4(1), 13- 39.

DÍAZ HUERTA, R. (2009). El blog: una estrategia reflexiva, creativa y propositiva en el proceso de enseñanza aprendizaje en el aula. II Jornadas de Innovación Docente en Tecnologías de la Información y Telecomunicaciones (ID+TIC). Alcalá de Henares - España: Escuela Superior Politécnica.

DODGE, B. (1995). Some thoughts about WebQuests. Consultado el 18 de abril de 2012. http://webquest.sdsu.edu/about_webquests.html

DREXLER, W., DAWSON, K. \& FERDIG, R.E. (2007). Collaborative Blogging as a Means to Develop Elementary Expository Writing Skills. Electronic Journal for the Integration of Technology in Education, 6, 140-160.

LINCOLN, Y.S. \& GUBA, E.G. (1985). Naturalistic Inquiry. Bervely Hills, CA: Sage.

MARTíN, M.A. y ALONSO, L. (2009). Los blogs y su utilidad en la enseñanza de la Historia de la Educación. XV Coloquio de Historia de la Educación, Pamplona-Iruñea, 29, 30 de junio y 1 de julio de 2009, 737-744.

MARTÍNEZ SÁNCHEZ, F. y PRENDES ESPINOSA, M.P. (2003). ¿A dónde va la educación en un mundo de tecnologías? En MARTíNEZ, F. (2003). Redes de comunicación en la enseñanza. Barcelona: Paidós.

MILES, M.B. \& HUBERMAN, A.M. (1994). An expanded sourcebook qualitative data analysis. Newbury Park, CA: Sage.

NAGEL, D.M. \& ANTHONY, K. (2009). Writing therapy using new technologiesthe art of blogging. Journal of Poetry Therapy: The Interdisciplinary Journal of Practice, Theory, Research and Education, 22 (1), 41-45.

NIELSEN, J. (2006). Participation Inequality: Encouraging More Users to Contribute. Alertbox, 1, 90-9.

PATTON, M.Q. (1990). Qualitative Evaluation and Research Methods (2nd ed.). Newbury Park, CA: Sage.

PÉREZ, F., ARANDA, E., hERNÁNDEZ, A., MARTíN, A., BENITO, M.J. y CóRdobA, M. de G. (2012). Otras actividades complementarias para mejorar la docencia universitaria: Elaboración y utilización de blogs. EDUTEC, Revista Electrónica de Tecnología Educativa, 40. 
Recuperado

el

$22 / 10 / 12$

de

http://edutec.rediris.es/Revelec2/Revelec40/actividades complementarias mejora docenci a unive rsitaria elaboracion y utilizacion blogs.html

RODERA BERMÚDEZ, A.M. (2008). Catalogación y valoración de las webquests desde el área de educación física y el tratamiento de temáticas transversales. Edutec, Revista Electrónica de Tecnología Educativa, 27. Consultado el 17/04/12. http://edutec.rediris.es/revelec2/revelec27/

TORRES ZUÑIGA, V. (2009). ¿Por qué las bitácoras electrónicas (blogs) se usan poco para estudiar ciencias físico-matemáticas? EDUTEC, Revista Electrónica de Tecnología Educativa, 29. Consultado el 17/04/12. http://edutec.rediris.es/revelec2/revelec29/

\section{Para citar este artículo:}

SANTOS, L.; \& FERNÁNDEZ, J. (2012).Uso pedagógico del blog: un proyecto de investigaciónacción en la materia de educación física en educación secundaria. EDUTEC, Revista Electrónica de Tecnología Educativa, 42. Recuperado el dd/mm/aa de http://edutec.rediris.es/Revelec2/Revelec42/uso pedagogico blog proyecto investigacionaccion.html 